\title{
CORRIGENDA
}

\section{The radiation dose-regulated AND gate genetic circuit, a novel targeted and real-time monitoring strategy for cancer gene therapy}

M Ding, E Zhang, R He, X Wang, R Li, W Wang and Q Yi

Cancer Gene Therapy (2012) 19, 592; doi:10.1038/cgt.2012.43

Correction to: Cancer Gene Therapy (2012) 19, 382-392; doi:10.1038/cgt.2012.11; published online 13 April 2012.

The authors' affiliations were published incorrectly. The author list with the correct affiliations appears below.

Miao Ding ${ }^{1}$; Erlong Zhang ${ }^{2}$; Rong $\mathrm{He}^{3}$; Xingyong $\mathrm{Wang}^{3}$; Rong $\mathrm{Li}^{2}$; Weidong Wang ${ }^{4}$ and Qijian $\mathrm{Yi}^{1}$
${ }^{1}$ Department of Cardiology and ${ }^{3}$ Emergency, Children Hospital, Chongqing Medical University, Chongqing, China; ${ }^{2}$ Institute of Combined Injury, State Key Laboratory of Trauma, Burns and Combined Injury, Chongqing Engineering Research Center for Nanomedicine, College of Preventive Medicine, Third Military Medical University, Chongqing, China; ${ }^{4}$ Department of Radiation Oncology, Tumor Hospital of Sichuan, Chengdu, Sichuan Province, China.

\section{Intratumoral delivery of CD154 homolog (Ad-ISF35) induces tumor regression: analysis of vector biodistribution, persistence and gene expression}

J Melo-Cardenas, M Urquiza, TJ Kipps and JE Castro

Cancer Gene Therapy (2012) 19, 592; doi:10.1038/cgt.2012.44

Correction to: Cancer Gene Therapy (2012) 19, 336-344; doi: 10.1038/cgt.2012.6

Information regarding an additional funding agency was missing from the Acknowledgment section. It should have included Gateway for Cancer Research Grant (JC, JMC, MU). The authors regret the error. 